\title{
Adverse events and treatment interruption in tuberculosis patients with and without HIV co- infection
}

\author{
R A M Breen, R F Miller, T Gorsuch, C J Smith, A Schwenk, W Holmes, J Ballinger, \\ L Swaden, M A Johnson, I Cropley, M C I Lipman
}

See end of article for authors' affiliations .......................

Correspondence to: Dr R A M Breen Department of Thoracic and HIV Medicine, Royal Free Hospital, London NW3 2QG, UK; r.breen@ medsch.ucl.ac.uk

Received 18 January 2006 Accepted 7 June 2006

Published Online First 14 July 2006
Background: Serious treatment associated adverse events are thought to occur more frequently in individuals with tuberculosis (TB) who are co-infected with HIV. A study was undertaken to assess the frequency of serious (grade III/IV) adverse events and interruption of anti-TB treatment in the era of effective antiretroviral therapy.

Methods: The incidence of serious adverse events was retrospectively compared in 312 individuals treated for TB, 156 of whom were co-infected with HIV.

Results: $111 \mathrm{HIV}$ infected individuals (71\%) received highly active antiretroviral therapy at the same time as anti-TB treatment. Serious adverse events were recorded in $40 \%$ HIV infected and $26 \%$ HIV uninfected individuals $(p=0.008)$. Peripheral neuropathy and persistent vomiting were more common in co-infected patients $(p<0.001 ; p=0.006)$, although all cause interruption of anti-TB treatment occurred with similar frequency in the two groups (13\% in HIV infected patients and $15 \%$ in HIV uninfected patients; $p=0.74$ ). In $85 \%$ of HIV infected patients and $87 \%$ of HIV uninfected individuals this was due to hepatotoxicity, which typically presented within 2 months of starting treatment. The median delay in restarting treatment was 4 weeks, so most individuals required full TB re-treatment.

Conclusion: Despite a greater rate of serious (grade III/IV) adverse events among HIV infected individuals, discontinuation of anti-TB treatment occurred with a similar frequency in HIV infected and HIV uninfected individuals.
A lthough the response to anti-tuberculosis (TB) treatment among HIV co-infected patients is generally good, many require concurrent highly active antiretroviral therapy (HAART) to achieve a successful long term outcome. $^{12}$ It has been reported that HIV/TB co-infected patients experience a higher rate of adverse drug reactions to treatment than those without HIV. ${ }^{3}$ Prior to the use of HAART, this may have reflected the additional toxicity of treatment for other opportunistic infections or that due to specific anti-TB agents such as thiacetazone. ${ }^{45}$ Few data exist in co-infected individuals since HAART has become widely available, although a very high rate of adverse drug reactions has been described. ${ }^{6}$ We hypothesised that, through careful selection of treatment regimens to minimise toxicity, the presence of HIV co-infection should have little impact on the frequency of serious (grade III/IV) adverse events, especially those causing treatment interruption, even if HAART was coadministered.

\section{METHODS}

Data were collected retrospectively at the North Middlesex, Royal Free, and University College London Hospitals, London. Consecutive unselected adult HIV infected individuals treated for TB between February 1997 and November 2003 were identified and compared with a control group of HIV uninfected individuals attending these treatment centres for TB treatment during the study period. Over this time the policy on testing for HIV infection of patients with TB changed from it being offered at physician discretion, based on an assessment of risk, to one of all patients being offered a test regardless of risk. To minimise possible selection bias we therefore included consecutive HIV uninfected individuals from the end of the study period working backwards in time until the two groups were of an identical size.

Tuberculosis was diagnosed if a patient had (1) a positive culture for Mycobacterium tuberculosis; or (2) was culture negative but nucleic acid amplification assay positive (TB Strand Displacement Amplification assay, Becton-Dickinson, New Jersey, USA) with clinicoradiological features and response to treatment consistent with TB; or (3) had histological findings and response to treatment consistent with TB.

Retrospective case note review identified the occurrence of serious (grade III/IV) adverse drug reactions and episodes of treatment interruption. Treatment interruption was defined as any period in which anti-TB therapy was discontinued due to an adverse drug reaction. Hepatotoxicity was defined as liver transaminases (AST/ALT) rising to $>5$ times the upper limit of normal, or a rising bilirubin level. ${ }^{7}$ Liver function was routinely checked at baseline (along with assessment of hepatitis B and C status) and repeated at 2 weeks. In individuals with normal test results, further blood tests were performed only in the event of new symptoms. In patients with chronic liver disease, liver function was checked every second week for 2 months and those with abnormal liver function before treatment or at week 2 were re-checked every second week until they had normalised or were clearly stable. HIV infected individuals with TB generally had liver function tests monitored more frequently throughout their treatment course, although the frequency of this was at the discretion of

Abbreviations: HAART, highly active antiretroviral therapy; NRTI, nucleoside reverse transcriptase inhibitor; NNRTI, non-nucleoside reverse transcriptase inhibitor; $\mathrm{Pl}$, protease inhibitor; $\mathrm{TB}$, tuberculosis 
the treating physician. Following discontinuation, treatment was recommenced according to national guidelines. ${ }^{7}$

Other adverse events were as documented by the treating clinician in the patient notes and graded by the investigators based on a standard classification system. ${ }^{8}$ The definitions of the most notable serious adverse events were: hepatotoxicity as described above; peripheral neuropathy as a marked decrease in sensation to the level of the knees or wrists; arthralgia as joint pain causing marked impairment of activities of daily living or mobility; persistent vomiting as vomiting of all food/fluids for 24 hours; and rash as vesiculation or moist desquamation or ulceration.

If anti-TB treatment had been discontinued, re-introduction was attempted sequentially in the order isoniazid, rifamycin, pyrazinamide, with the dose of each agent increased up to the therapeutic amount over 2 or 3 days with a period of 2 or 3 days of observation between restarting each agent. ${ }^{7}$ Data were recorded on a standard proforma and entered on a central database.

Prescription of HAART and anti-TB treatment was at the discretion of individual physicians using the treatment protocols then available. In all cases initial treatment of TB was rifamycin based and was adjusted according to availability of mycobacterial susceptibility testing. A typical regimen would start with a rifamycin plus isoniazid and pyrazinamide (ethambutol was routinely commenced at North Middlesex and Royal Free but not University College) for 2 months followed by a continuation phase of two drugs dependent on drug sensitivities. All patients receiving isoniazid were co-administered pyridoxine 10$25 \mathrm{mg}$ daily. Standard dose rifampicin $(600 \mathrm{mg}$ if $>50 \mathrm{~kg}$, $450 \mathrm{mg}$ if $<50 \mathrm{~kg}$ ) was prescribed except with single or "boosted" protease inhibitor containing regimens when rifabutin was given ( $150 \mathrm{mg}$ daily or $150 \mathrm{mg}$ three times per week, respectively). HAART was defined as use of $\geqslant 3$ antiretroviral drugs in combination. Standard practice was to prescribe all drugs as daily self-administered treatment.

\section{Analysis of data}

Comparisons between groups were performed using either the $\chi^{2}$ test or Fisher's exact test. The data were analysed using SAS version 8.2 (SAS Institute Inc, Cary, NC, USA).

\section{RESULTS}

One hundred and fifty six HIV infected individuals were treated for TB during the study period (TB+HIV+). The characteristics of these individuals and of a control group of

Table 1 Comparison of HIV infected individuals with TB and a control group of HIV uninfected patients with TB

\begin{tabular}{lll}
\hline & $\begin{array}{l}\text { HIV infected } \\
(\mathbf{n}=156)\end{array}$ & $\begin{array}{l}\text { HIV uninfected } \\
(\mathbf{n}=156)\end{array}$ \\
\hline $\begin{array}{l}\text { Median (range) age (years) } \\
\text { Male sex }\end{array}$ & $35(20-73)$ & $33(16-82)$ \\
Ethnicity & $81(52 \%)$ & $90(58 \%)$ \\
$\quad$ Black African & $122(78 \%)$ & $64(41 \%)^{*}$ \\
$\quad$ Asian & $3(2 \%)$ & $45(28 \%)$ \\
$\quad$ White & $28(18 \%)$ & $47(30 \%)$ \\
$\quad$ Other & $3(2 \%)$ & $0(0 \%)$ \\
Pulmonary TB & $89(57 \%)$ & $80(51 \%)$ \\
Median (range) duration of & $6(1-24)$ & $6(1-36)$ \\
anti-TB treatment (months) & $9(6 \%)$ & $6(4 \%)$ \\
Discontinuation of anti-TB & 9 treatment & $2(1 \%)$ \\
Incidence of TB recurrence & $5(3 \%)$ & $5(3 \%)$ \\
Incidence of hepatitis B or C & $5(3 \%)$ & \\
co-infection & & \\
\hline * $\mathrm{p}<0.0001$. & &
\end{tabular}

156 HIV uninfected individuals with tuberculosis (TB+HIV-) are shown in table 1.

In the TB+HIV+ group, the median CD4 count and HIV load at the time of starting anti-TB treatment was 77 (range $0-720$ ) cells/ $\mu$ l and $5.1 \log _{10}$ (range $1.7-5.9$ ) viral copies/ml respectively. One hundred and eleven of the $156 \mathrm{HIV}$ infected individuals $(71 \%)$ received HAART while on anti-TB treatment; 29 (26\%) were already using HAART when anti-TB treatment was started and the remaining 82 commenced HAART a median of 2 (range $0-8$ ) months later.

Treatment interruptions and common serious (grade III/ IV) adverse events are described in table 2. Serious adverse events occurred in 63 of $156(40 \%)$ TB+HIV+ individuals and in 41 of $156(26 \%)$ TB+HIV - individuals $(p=0.008)$. The most frequent adverse event in TB+HIV+ individuals was peripheral neuropathy, which occurred in $22(14 \%)$ compared with $3(2 \%)$ of those without HIV co-infection $(\mathrm{p}<0.0001)$. Of these $22 \mathrm{~TB}+\mathrm{HIV}+$ individuals, 17 were receiving concomitant HAART and 11 used regimens containing the nucleoside reverse transcriptase inhibitors (NRTI) stavudine (d4T) and/or didanosine (ddI). However, excluding those who received ddI and/or d4T in whom the only adverse event was peripheral neuropathy, the overall frequency of adverse events was little changed at $38 \%$. In both TB populations hepatotoxicity developed in 20 (13\%) of 156 individuals, all of whom were symptomatic. Only one case of hepatotoxicity occurred in an individual with hepatitis B co-infection and none were associated with hepatitis C co-infection.

Rash occurred frequently and with a similar incidence in both groups. Persistent vomiting was significantly more common among TB+HIV+ individuals $(\mathrm{p}=0.006)$, and a greater number of $\mathrm{TB}+\mathrm{HIV}+$ individuals had more than one serious adverse event than in the TB+HIV- population $(\mathrm{p}=0.02$, table 2$)$.

Interruption of anti-TB treatment occurred in $13 \%$ of $\mathrm{TB}+\mathrm{HIV}+$ individuals and $15 \%$ of $\mathrm{TB}+\mathrm{HIV}-$ individuals $(p=0.74)$. In TB+HIV+ individuals, 17 of $20(85 \%)$ interruptions were secondary to hepatotoxicity. Other causes were rash $(n=2)$ and peripheral neuropathy $(n=1)$. Among the TB+HIV - individuals, 20 of $23(87 \%)$ interruptions were secondary to hepatotoxicity; the other three were due to thrombocytopenia, vomiting, and optic neuritis.

In both $\mathrm{TB}+\mathrm{HIV}+$ and $\mathrm{TB}+\mathrm{HIV}-$ groups almost all interruptions in anti-TB treatment occurred within the first 2 months. There was a median delay of 4 weeks before full anti-TB treatment could be restarted. All such interruptions lasted for at least 1 week. HAART was discontinued in 11 of $111 \mathrm{~TB}+\mathrm{HIV}+$ individuals $(10 \%)$ while they were receiving anti-TB treatment (four due to HAART related adverse events (three had immune reconstitution inflammatory syndrome) and seven due to virological failure). In a further 12 individuals $(11 \%)$ the HAART regimen was altered during the course of anti-TB treatment.

Comparison of the frequency of both adverse events and interruptions in anti-TB treatment in the black African population showed that these were twice as high in the HIV+TB+ group as in the TB+HIV - group (43\% v 21\% for adverse events $(p=0.07)$; $13 \% \vee 6 \%$ for treatment interruption $(p=0.03))$. In the white population the frequency of adverse events and treatment interruptions were similar in those with and without HIV co-infection (38\% v 32\% for adverse events; $17 \% v 19 \%$ for treatment interruption). It was not possible to perform a similar comparison in the Asian population as the number of HIV infected subjects was very small. Asian TB+HIV - individuals showed high rates of both adverse events (33\%) and interruptions in anti-TB treatment (22\%; all due to hepatotoxicity).

In the $82 \mathrm{HIV+TB}+$ individuals who started HAART after anti-TB treatment, 32 did so within 2 months (median 
Table 2 Incidence of serious (grade III or IV) adverse events (AE) during the treatment of TB according to HIV status

\begin{tabular}{llll}
\hline & $\begin{array}{l}\text { HIV infected } \\
(\mathbf{n}=156)\end{array}$ & $\begin{array}{l}\text { HIV uninfected } \\
(\mathbf{n}=156)\end{array}$ & p value \\
\hline Individuals with at least one AE & $63(40 \%)$ & $41(26 \%)$ & 0.008 \\
Hepatotoxicity & $20(13 \%)$ & $20(13 \%)$ & 1.00 \\
Peripheral neuropathy & $22(14 \%)$ & $3(2 \%)$ & $<0.0001$ \\
Rash & $20(13 \%)$ & $13(8 \%)$ & 0.20 \\
Persistent vomiting & $15(10 \%)$ & $3(2 \%)$ & 0.006 \\
Arthralgia & $6(4 \%)$ & $1(1 \%)$ & 0.07 \\
Individuals with more than one AE & $11(7 \%)$ & $2(1 \%)$ & 0.02 \\
Individuals requiring interruption of anti-TB treatment & $20(13 \%)$ & $23(15 \%)$ & 0.74 \\
\hline & & & \\
\hline
\end{tabular}

(range) CD4 count $40(10-390)$ cells $/ \mu \mathrm{l})$ and 50 started after 2 months (median (range) CD4 count 52 (10-400) cells/ $\mu \mathrm{l})$. The frequency of adverse events in those starting HAART within 2 months and after 2 months was 53\% and $48 \%$, respectively; treatment interruptions occurred in $16 \%$ and $18 \%$, respectively. No differences were noted in the occurrence of adverse events or treatment interruption whether HAART was started before anti-TB treatment, within or after 2 months of starting anti-TB treatment, or not at all ( $p=0.73$ for adverse events; $\mathrm{p}=0.55$ for interruption). In this group 56 of 82 had a CD4 count at TB diagnosis of $<100$ cells/ $\mu l$, and the frequency of adverse events and treatment interruptions in these individuals was $52 \%$ and $16 \%$, respectively. Twenty six of 82 had a CD4 count at TB diagnosis of $>100$ cells/ $\mu \mathrm{l}$ with a frequency of adverse events and treatment interruptions in these individuals of $48 \%$ and $22 \%$, respectively $(p=0.74$ for adverse events; $p=0.84$ for treatment interruption).

Five HAART regimens were prescribed (involving 31 different combinations of drugs). The five regimens were: nucleoside reverse transcriptase inhibitor (NRTI); two or three NRTI + non-nucleoside reverse transcriptase inhibitor (NNRTI); two or three NRTI + single protease inhibitor (PI); two or three NRTI + boosted PI; and two or three NRTI + NNRTI + PI (table 3). The frequency of adverse events, hepatotoxicity, and interruptions in anti-TB treatment were assessed in the $111 \mathrm{~TB}+\mathrm{HIV}+$ individuals according to the regimen of HAART prescribed. No differences were seen in the frequency of events with different regimens, although the numbers in some groups were small.

\section{DISCUSSION}

Our data show that, despite the fact that treatment of TB was associated with a greater frequency of serious (grade III/IV) adverse events in HIV co-infected individuals than in patients with TB alone $(40 \% \vee 26 \%)$, this did not translate into an increased frequency of treatment interruptions (which occurred in approximately one in seven subjects). These findings did not appear to be altered by the level of immunosuppression, the type of HAART regimen prescribed, or its time of initiation.

The most common reason for interrupting anti-TB treatment was hepatitis. This occurred mainly within the first 2 months of treatment, with a median time off anti-TB treatment of 4 weeks. Thus, most interruptions necessitated full re-treatment. In contrast with other reports, we found this to occur at a similar frequency in both HIV infected and uninfected patients. ${ }^{9}$ The difference in study results may reflect the criteria used to perform liver function tests, as well as the point at which treatment would be discontinued given an abnormal result. We used national guidelines which combine both blood test results and symptoms in deciding whether to repeat liver function tests. ${ }^{7}$ These are formulated primarily for HIV uninfected TB individuals, and it is likely that HIV infected individuals with TB tend to undergo more frequent blood tests.

Previous studies have identified a number of risk factors for anti-TB treatment induced hepatitis including disease extent, chronic hepatitis, and alcohol use. ${ }^{9-12}$ Both groups had a low prevalence of chronic viral hepatitis which made assessment of this as a risk factor unfeasible. We did not assess the extent of disease but sought to minimise the possibility that, early in the study, only the sickest patients were tested for HIV by identifying the HIV uninfected cohort in reverse time order. In most cases the use of alcohol, recreational drugs, and over-the-counter preparations was poorly recorded in the notes, and we felt that findings based on incomplete data might be misleading.

Only in the black African population did ethnicity appear to be important, with a significantly lower frequency of antiTB treatment interruption observed in the HIV uninfected group than in the HIV infected group. The effect of ethnicity in this area is not well described but our data suggest that it may be important. Ethnicity may be one explanation for the marked difference in the observed frequency of hepatotoxicity necessitating treatment interruption in our series, compared with $3.2 \%$ in the UK study by Ormerod and Horsfield ${ }^{13}$ in which $70 \%$ of the subjects were Asian.

Table 3 Incidence of serious (grade III or IV) adverse events, hepatotoxicity, and interruptions in anti-TB treatment according to HAART regimen $(n=111)$

\begin{tabular}{lllll}
\hline Type of HAART regimen & $\begin{array}{l}\text { No of patients } \\
\text { receiving regimen }\end{array}$ & $\begin{array}{l}\text { Serious adverse } \\
\text { events }\end{array}$ & $\begin{array}{l}\text { Interruptions to anti-TB } \\
\text { treatment }\end{array}$ \\
\hline Triple NRTI & 9 & $5(56 \%)$ & $2(22 \%)$ & $2(22 \%)$ \\
$2 \mathrm{NRTI}+\mathrm{NNRTI}$ & 69 & $31(45 \%)$ & $9(13 \%)$ & $8(12 \%)$ \\
$2 \mathrm{NRTI}+\mathrm{PI}$ & 11 & $5(45 \%)$ & $2(18 \%)$ & $2(18 \%)$ \\
$2 \mathrm{NRTI}+$ "boosted" PI & 13 & $5(38 \%)$ & $0(0 \%)$ & $0(0 \%)$ \\
$2 \mathrm{NRTI}+\mathrm{PI}+\mathrm{NNRTI}$ & 5 & $2(40 \%)$ & $1(20 \%)$ & $1(20 \%)$ \\
Not recorded & 4 & $0(0 \%)$ & $0(0 \%)$ & $0(0 \%)$ \\
\hline
\end{tabular}

NRTI, nucleoside reverse transcriptase inhibitor; NNRTI, non-nucleoside reverse transcriptase inhibitor; PI, protease inhibitor. 
An earlier study of the treatment of HIV/TB co-infection in the era of HAART observed an incidence of significant adverse events of more than $50 \%$ with one third of subjects discontinuing anti-TB treatment. ${ }^{6}$ However, it did not contain an HIV uninfected comparator population which makes it difficult to assess the relative contributions of HIV, HAART and anti-TB treatment.

A large proportion of the excess adverse event rate in HIV/ TB co-infected individuals was due to peripheral neuropathy. We expected some of this to result from co-administration of $\mathrm{d} 4 \mathrm{~T}$ or ddI with isoniazid, which is now not recommended due to a reported increase in neuropathy. ${ }^{14} 15$ However, removal of those who received $\mathrm{d} 4 \mathrm{~T} / \mathrm{ddI}$ and isoniazid from the analysis did not substantially reduce the observed incidence, which suggests the importance of other factors such as HIV itself. ${ }^{16}$

The retrospective collection of data meant that some adverse events may not have been recorded. However, rates of under-reporting should have been similar regardless of HIV status. Adherence was not formally assessed but we believe that it was generally very good-reflected in the high rates of treatment completion, a low rate of relapse of TB, and the good virological response to HAART (reported elsewhere $\left.{ }^{17}\right)$. Other limitations of our study include potential bias due to ethnic mix, difficulties in assessing the extent of TB disease as well as alcohol and other drug use, and the different frequency of blood test monitoring between groups.

In conclusion, our data suggest that, despite a higher frequency of serious (grade III/IV) adverse events during anti-TB treatment in individuals with HIV co-infection, treatment interruption occurs no more frequently than in HIV uninfected patients with TB.

\section{Authors' affiliations}

R Breen, W Holmes, J Ballinger, L Swaden, M A Johnson, I Cropley,

M C I Lipman, Department of HIV Medicine, Royal Free Hospital, London, UK

R F Miller, C J Smith, Department of Primary Care and Population Sciences, Royal Free and University College Medical School, London, UK T Gorsuch, A Schwenk, Department of HIV Medicine, North Middlesex Hospital, London, UK
Funding: none.

Competing interests: none declared.

\section{REFERENCES}

1 Chaisson RE, Schecter GF, Theurer CP, et al. Tuberculosis in patients with the acquired immunodeficiency syndrome: clinical features, response to therapy, and survival. Am Rev Respir Dis 1987;136:570-4.

2 Dheda K, Lampe F, Johnson MA, et al. Outcome of HIV-associated tuberculosis in the era of highly active antiretroviral therapy. $J$ Infect Dis 2004; 190:1670-6.

3 Yee D, Valiquette $C$, Pelletier $M$, et al. Incidence of serious side effects from first-line antituberculosis drugs among patients treated for active tuberculosis. Am J Respir Crit Care Med 2003;167:1472-7.

4 Pedral-Sampaio DB, Martins Netto E, Alcantara AP, et al. Use of standard therapy for tuberculosis is associated with increased adverse reactions in patients with HIV. Braz J Infect Dis 1997;1:123-30.

5 Nunn $P$, Kibuga $D$, Gathua $S$, et al. Cutaneous hypersensitivity reactions due to thiacetazone in HIV-1 seropositive patients treated for fuberculosis. Lancet 1991;337:627-30.

6 Dean GL, Edwards SG, Ives NJ, et al. Treatment of tuberculosis in HIVinfected persons in the era of highly antiretroviral therapy. AIDS 2002;16:75-83.

7 Joint Tuberculosis Committee of the British Thoracic Society. Chemotherapy and management of tuberculosis in the United Kingdom: recommendations 1998. Thorax 1998;53:536-48

8 AIDS Clinical Trials Group. Table for grading severity of adult adverse experiences. Rockville, MD: Division of AIDS, National Institute of Allergy and Infectious Diseases, 1996.

9 Ungo JR, Jones D, Ashkin D, et al. Anti-tuberculosis drug-induced hepatoxicity. The role of hepatitis $C$ and the human immunodeficiency virus. Am J Respir Crit Care Med 1998;157:1871-6.

10 Wong WM, Wu PC, Yuen MF, et al. Antituberculosis drug-related liver dysfunction in chronic hepatitis B infection. Hepatology 2000;31:201-6.

11 Pande JN, Singh SP, Khilnani GC, et al. Risk factors for hepatotoxicity from antituberculosis drugs: a case-control study. Thorax 1996;51:132-6.

12 Dossing M, Wilcke JT, Askgaard DS, et al. Liver injury during antituberculosis treatment: an 11-year study. Tuberc Lung Dis 1996;77:335-40.

13 Ormerod LP, Horsfield N. Frequency and type of reactions to antituberculosis drugs: observations in routine treatment. Tuberc Lung Dis 1996;77:37-42.

14 Breen RA, Lipman MC, Johnson MA. Increased incidence of peripheral neuropathy with co-administration of stavudine and isoniazid in HIV-infected individuals. AIDS 2000;14:615.

15 Pozniak AL, Miller RF, Lipman MC, et al. BHIVA Guidelines Writing Committee. BHIVA guidelines for tuberculosis (TB)/HIV infection 2005. HIV Med 2005; Suppl 2:62-83.

16 Barohn RJ, Gronseth GS, LeForce BR, et al. Peripheral nervous system involvement in a large cohort of human immunodeficiency virus-infected individuals. Arch Neurol 1993;50:167-71.

17 Breen RAM, Miller RF, Gorsuch T, et al. Virological response to HAART is unaffected by anti-tuberculosis therapy. J Infect Dis 2006;193:1437-40. 